\title{
Enhancer of zeste homolog 2 blockade by RNA interference is implicated with inhibited proliferation, invasion and promoted apoptosis in endometrial carcinoma
}

\author{
JUAN WANG, ZHIHONG AI, JING CHEN, YINCHENG TENG and JIEPING ZHU \\ Department of Obstetrics and Gynecology, The Sixth People's Hospital, \\ Shanghai Jiao Tong University, Shanghai 200233, P.R. China
}

Received March 31, 2016; Accepted December 8, 2017

DOI: $10.3892 / \mathrm{ol} .2018 .8518$

\begin{abstract}
Endometrial carcinoma is the most common gynecological malignancy of the female genital tract worldwide (2012). Enhancer of zeste homolog 2 (EZH2), a critical component of the polycomb repressive complex 2 , has been found to be associated with multiple biological processes and is overexpressed in multiple types of cancer. Previous studies have demonstrated that EZH2 is associated with endometrial carcinoma. The present study investigated the expression and biology function of EZH2 in endometrial cancer (EC). It was found that EZH2 levels were markedly increased in endometrial cancer tissues compared with that in adjacent normal tissues. EZH2 was significantly overexpressed in 3 separate endometrial cancer cell lines (Ishikawa, RL95-2 and HEC1-A) when compared with the normal endometrial cell line ESC. Additionally, small interfering RNA was used to investigate the role of EZH2 in endometrial carcinoma cell proliferation, and the results showed that EZH2 knockdown suppressed the proliferation of endometrial carcinoma cells in vitro. Furthermore, EZH2 knockdown induced apoptosis of human EC cells by promoting the expression of pro-apoptosis protein caspase 3 , caspase 9, BCL2 associated $X$ and decreasing the expression of anti-apoptosis protein Bcl-2. Finally, the present study demonstrated that EZH2 knockdown suppressed the invasion of EC cells through downregulation of the epithelial-mesenchymal transition. Collectively, these data demonstrate that EZH2 is frequently overexpressed in EC cells and its overexpression is associated with promoting the proliferation and invasion and decreasing the apoptosis of EC cells, suggesting that EZH2 may provide potential therapeutic targets for treatment of endometrial carcinoma.
\end{abstract}

Correspondence to: Dr Jieping Zhu, Department of Obstetrics and Gynecology, The Sixth People's Hospital, Shanghai Jiao Tong University, 600 Yishan Road, Shanghai 200233, P.R. China

E-mail: jpzhuzhu@yeah.net

Key words: enhancer of zeste homolog 2, endometrial cancer, proliferation, invasion, apoptosis

\section{Introduction}

Endometrial carcinoma (EC) is the most common gynecological malignancy, and its prevalence is increasing annually (1). EC is often diagnosed at an early stage to the presence of various symptoms, including vaginal bleeding, pain with urination, pain during sexual intercourse or pelvic pain (2). However, a smaller proportion of patients with EC are diagnosed at advanced stage accompanied by malignant proliferation, extensive invasion, and lymphatic metastasis or some patients develop disease recurrence (3). The prognosis for advanced stage or recurrent patients is still poor and the mortality is high (4). Therefore, improved understanding of the pathogenesis and identification of the molecular alterations is urgent for the identification of novel therapeutic targets that assist novel effective therapies for endometrial cancer.

Enhancer of zeste homolog $2(\mathrm{EZH} 2)$ is a member of the polycomb group of genes $(\mathrm{PcG})$ and serves as a histone methyltransferase that mediates target genes silencing by trimethylating the lysine 27 of histone H3 (5). EZH2 has been found to be associated with multiple biological processes, including tumor proliferation, cell cycle regulation, cell fate determination, cell differentiation, senescence, metastasis and angiogenesis (6). Mounting evidence demonstrates that EZH2 is overexpressed or mutant in aggressive forms of prostate cancer (7), breast cancer (8), bladder cancer (9), non-small cell lung carcinoma (10) and ovarian cancer (11) and is associated with poor prognosis in multiple types of cancer.

EZH2 expression is associated with a high proliferation rate and aggressive tumor subgroups of endometrial cancer (12). EZH2 expression has been demonstrated to be positively associated with the expression of lipocalin 2 (13) and focal adhesion kinase (FAK) (14), which is associated with aggressive features of endometrial cancer. EZH2 expression is associated with tumor cell proliferation, migration, and invasion in endometrial cancer cell lines as well as with increased stage, grade, depth of invasion, and nodal metastasis in human cancer tissue specimens, which is parallel to an increased expression of Wnt pathway inhibitors and a concomitant decrease in $\beta$-catenin levels (15).

Although EZH2 expression is associated with the proliferation and aggressive feature of endometrial cancer $(12,15,16)$, 
its function in EC is poorly elucidated, especially for the underlying mechanism. In this article, we examined the potential role of EZH2 on EC cell line proliferation, apoptosis, and invasion.

\section{Materials and methods}

Cell culture. Human endometrial cancer, RL95-2, HEC1-A, Ishikawa cells and the normal human endometrial cell line ESC, as negative control cells, were purchased from American Type Culture Collection (Manassas, VA, USA). HEC-1 and Ishikawa cells were maintained in Mcoy's 5A media supplemented with $10 \%$ fetal bovine serum (FBS; Hyclone, Logan, UT, USA); ESC cells were cultured in a phenol-free DMEM-F12 1:1 mixture supplemented with $1 \%$ ITS liquid media supplement (Sigma-Aldrich, St. Louis, MO, USA) + premix, and 10\% charcoal treated FBS; RL95-2 cells were grown in DMEM-F12medium supplemented with $10 \%$ FBS and $0.005 \mathrm{mg} / \mathrm{ml}$ insulin (Sigma-Aldrich; Merck KGaA, Darmstadt, Germany). All cells were supplemented with $1 \%$ penicillin and streptomycin (Thermo Fisher Scientific, Inc., Waltham, MA, USA) in humidified air at $37^{\circ} \mathrm{C}$ with $5 \% \mathrm{CO}_{2}$. All cell lines were tested and authenticated by DNA (short tandem repeat genotyping) profiling before use.

Tissue samples. A total of 40 endometrial tissues (including 20 endometrial cancer samples and 20 adjacent normal endometrial tissues as negative controls) were obtained from patients who underwent surgery and for immunohistochemical analysis at the Sixth People's Hospital of Shanghai Jiao Tong University. All specimens were frozen immediately in liquid nitrogen and stored at $-80^{\circ} \mathrm{C}$ until RNA extraction. All specimens were reviewed by two pathologists from the Department of Obstetrics and Gynecology, The Sixth People's Hospital, Shanghai Jiao Tong University (Shanghai, China). None of the patients had received preoperative radiotherapy, chemotherapy, or hormonal therapy. All patients provided written informed consent permitting the use of their tissue for research. The present study has received Scientific and Ethical Committee approval from the institutional review board of the Sixth People's Hospital of Shanghai Jiao Tong University.

Quantitative reverse transcription-polymerase chain reaction $(R T-q P C R)$ analysis. Total RNA was isolated from cancerous/noncancerous specimens or cell lines with TRIzol reagents (Invitrogen; Thermo Fisher Scientific, Inc.). Total RNA $(1 \mu \mathrm{g})$ was reverse transcribed into cDNA using the PrimeScript RT reagent kit (TaKaRa Biotechnology, Shiga, Japan) under the following conditions; $37^{\circ} \mathrm{C}$ for $15 \mathrm{~min}$ and $85^{\circ} \mathrm{C}$ for $5 \mathrm{sec}$. RT-qPCR was performed according to the manufacturer's protocol of the SYBR Premix Ex Taq kit (Takara Biotechnology Co., Ltd., Dalian, China) on an Mx3005P thermal cycler (Stratagene; Agilent Technologies, Inc., Santa Clara, CA, USA). The PCR conditions were 25-30 cycles at $95^{\circ} \mathrm{C}$ for $30 \mathrm{sec}, 56^{\circ} \mathrm{C}$ for $30 \mathrm{sec}$, and $72^{\circ} \mathrm{C}$ for $1 \mathrm{~min}$. All reactions were performed in triplicate. The $2^{-\Delta \Delta \mathrm{Cq}}$ method was used to calculate the relative expression of each gene relative to the amount of GAPDH (17). The PCR primers used are as follows: EZH2 forward, 5'-TTGTTGGCGGAA
GCGTGTAAAATC-3' and reverse, 5'-TCCCTAGTCCCG CGCAATGAGC-3'; GAPDH forward, 5'-GTCAACGGA TTTGGTCTGTATT-3' and reverse, 5'-AGTCTTCTGGGT GGCAGT GAT-3'. All primers were obtained from Invitrogen (Thermo Fisher Scientific, Inc).

Western blot analysis. Cells were lysed in RIPA buffer (150 mM NaCl, $50 \mathrm{mM}$ Tris-base, $5 \mathrm{mM}$ EDTA, $1 \%$ NP-40, $0.25 \%$ deoxycholate, $\mathrm{pH}$ 7.4) with protease and phosphatase inhibitors (Roche Applied Science, Penzberg, Germany). Protein concentrations were measured using the BCA protein assay (cat. no. 23227; Thermo Fisher Scientific, Waltham, MA, USA). Equal amounts (30-50 $\mu \mathrm{g})$ of the protein were electrophoresed using SDS-PAGE (10\% gel), transferred to nitrocellulose membranes and incubated with the following primary antibodies overnight at $4{ }^{\circ} \mathrm{C}$ : Anti-EZH 2 antibody, (cat. no. 5246S, Cell Signaling Technology); anti- $\beta$-actin antibody, (Cell Signaling Technology); rabbit polyclonal anti-E-cadherin (cat. no. A01589, GenScript, Edison, NJ), mouse monoclonal anti-N-cadherin (BD, Transduction, San Jose, CA), rabbit polyclonal anti-Vimentin (cat. no. A01189, GenScript, Edison, NJ). Anti-Bax (cat. no. ab32503), anti-Bcl-2 (cat. no. ab117115), anti-caspase-3 (cat. no. ab2302), anti-caspase-9 (cat. no. ab52298) were purchased from Abcam. The primary antibody incubation was followed by incubation with goat anti-rabbit immunoglobulin (Ig)G-horseradish peroxidase (HRP; cat. no. sc-2004; 1:2,000, dilution), goat anti-mouse IgG-HRP (cat. no. sc-2005; 1:2,000, dilution) or rabbit anti-goat IgG-HRP (cat. no. sc-2922; 1:2,000, dilution) from Santa Cruz Biotechnology, Inc. (Dallas, TX, USA) for $1 \mathrm{~h}$ at room temperature. The bound antibodies were detected using the Enhanced Chemiluminscence western blotting substrate kit (cat. no. K820-50; BioVision, Inc., Milpitas, CA, USA).

EZH2 small interfering (si)RNA transfection. HEC-1A and RL95-2 cells were plated on 6-well plates at a density of $2 \times 10^{5}$ cells/well and cultured in corresponding mediums previously described overnight at $37^{\circ} \mathrm{C}$ until $30-40 \%$ confluency. The cells were transfected with three validated siRNA for EZH2 (si-EZH2-1: 5'-CCTGGTCTGGCTTTATGCTAA GTTT-3'; si-EZH2-2: 5'-TCGAGCTGCTCTGCTCTCTAT TGAT-3'; si-EZH2-3: 5'-GCTCTCTATTGATTGTGTTTC TGGA-3'; si-Control: 5'-CCTGTCTTTCGGTATAATCGG GTTT-3') and a scramble siRNA-FAM (negative control; Invitrogen) at a concentration of $100 \mathrm{nM}$. Using Lipofectamine 2000 transfection reagent (Invitrogen) according to the manufacturer's protocol. The medium was replaced with standard culture medium $6 \mathrm{~h}$ post-transfection. Transfection was repeated $72 \mathrm{~h}$ after the initial transfection.

In vitro cell proliferation assay. Cell proliferation was assayed using a cell proliferation kit, Cell Counting kit-8 (CCK-8; Dojindo Molecular Technologies, Inc., Kyushu, Japan) according to the manufacturer's protocol. HEC-1A and RL95-2 cells were seeded into 96-well tissue culture plates at a density of $2 \times 10^{3}$ cells/well the day before EZH2 siRNA transfection. Cell proliferation was analyzed at a wavelength of $450 \mathrm{~nm}$ at 24, 48, 72, 96 and $120 \mathrm{~h}$ after transfection using Envision (PerkinElmer, Inc., Waltham, MA, USA). Experiments were 

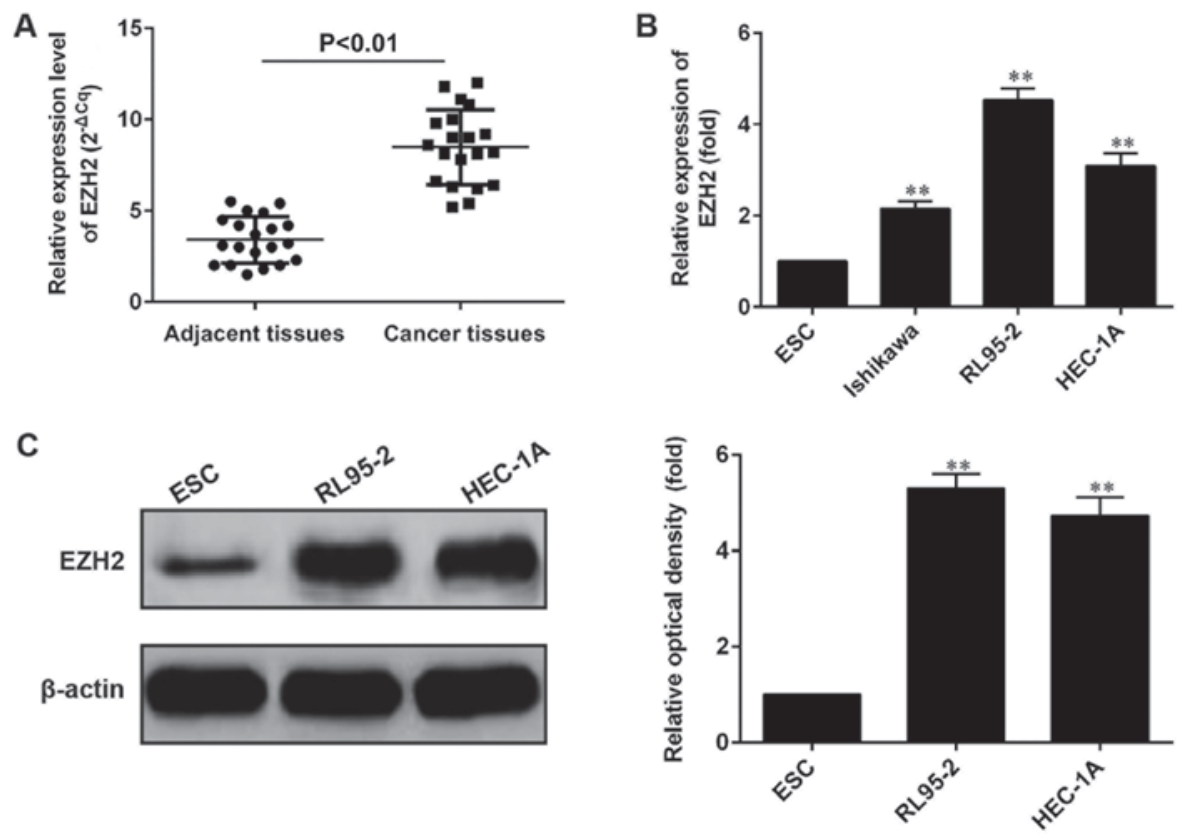

Figure 1. Relative expression of EZH2 in endometrial cancer tissues and cell lines. (A) Relative EZH2 expression in endometrial cancer tissues (n=20) in comparison with the corresponding adjacent normal tissues ( $\mathrm{n}=20 ; \mathrm{P}<0.01$ ). (B) Relative EZH2 expression in 4 endometrial cancer cell lines (Ishlkawa, RL95-2 and HEC-1A) in comparison with the normal endometrial cell line ESC. (A and B) EZH2 expression were examined by RT-qPCR and normalized to GAPDH expression. (C) The expression of EZH2 in the ESC, HEC-1A and RL95-2 cell lines was further examined by western blot analysis (right). The density of protein bands was quantified by using Image $\mathbf{J}$ software (left). Results shown are from 3 independent experiments. The ESC cell line was used as negative control. ${ }^{* *} \mathrm{P}<0.01$ vs. the negative group. EZH2, enhancer of zeste homolog 2; RT-qPCR, quantitative reverse transcription-polymerase chain reaction.

performed in triplicate. Cells transfected with siRNA-FAM were used as a negative control.

Annexin V/propidium iodide (PI) staining for detecting apoptotic cells. Cell apoptosis was determined by an Annexin V/PI assay according to the protocol of the Annexin V-FITC apoptosis kit (BD, Carlsbad, CA, USA). The Annexin V-FITC and PI fluorescence levels were measured by flow cytometry (BD Biosciences, FACS Calibur). The Annexin V-positive cells (PI-negative and -positive) were defined as apoptotic cells. Cells transfected with siRNA-FAM were used as a negative control.

Matrigel invasion assay. BD BioCoat ${ }^{\mathrm{TM}}$ Matrigel $^{\mathrm{TM}}$ Invasion Chamber was used to measure cell invasion according to the manufacturer's protocol. Cells $\left(1 \times 10^{5}\right.$ cells/well) suspended in $0.5 \mathrm{ml}$ medium were added to the upper compartment of 24-well Matrigel-coated or non-coated 8 micron membrane and medium supplemented with $10 \%$ FBS was applied to the lower compartment. After incubation for $22 \mathrm{~h}$ at $37^{\circ} \mathrm{C}$ and $5 \% \mathrm{CO}_{2}$, the cells were fixed with $4 \%$ formaldehyde at $37^{\circ} \mathrm{C}$ for $15 \mathrm{~min}$ and stained with $1 \%$ crystal violet in PBS at $37^{\circ} \mathrm{C}$ for $30 \mathrm{~min}$. The number of cells that migrated across the control membrane or invaded through the Matrigel-coated membrane was determined in 9 fields across the center and the periphery of the membrane. Cells transfected with siRNA-FAM were used as negative controls.

Statistical analysis. All assays were conducted on triplicate samples in triplicate. The data are presented as the mean \pm standard deviation. Statistical analyses were conducted using one-way analysis of variance with Tukey's post-hoc testing or unpaired student t-test using Prism 5.0 software (GraphPad Software, Inc., La Jolla, CA, USA). P $<0.05$ was considered to indicate a statistically significant difference.

\section{Results}

EZH2 is overexpressed in endometrial cancer. The level of EZH2 was detected in 20 paired endometrial cancer samples and adjacent histological normal tissues by RT-qPCR and normalized to GAPDH. In cancerous tissues, EZH2 expression was at a level significantly increased compared with the mean level of normal specimens ( $\mathrm{P}<0.01$; Fig. 1A). Expression of EZH2 was further examined by RT-qPCR in 3 separate endometrial cancer cell lines (Ishikawa, RL95-2 and HEC1-A) as well as the normal endometrial cell line ESC. When compared with ESC cells, EZH2 was expressed at increased levels (2-5 folds) in all cancer cell lines (Fig. 1B). We further confirmed the expression of EZH2 by Western Blot in HEC-1A and RL95-2 cancer cells (Fig. 1C). The expression of EZH2 was also at increased levels (4-6 folds), consistent with Fig. 1A and Fig. 1B.

EZH2 knockdown inhibits the growth of human EC cells in vitro. There was a significant correlation between EZH2 expression and Ki67 expression $(12,18)$. Therefore, the present study sought to examine the effects of EZH2 knockdown on proliferation of human EC cells. To knockdown EZH2 expression in EC cell lines (HEC-1A and RL95-2), we developed 3 individual siEZH2. The knockdown efficacy of siEZH2 in EC cell lines was confirmed by RT-qPCR (Fig. 2A). Compared with controls, EZH2 knockdown significantly decreased cell proliferation in the 2, 3, 4, 5 days as in indicated by CCK8 assays (Fig. 2B). 
A

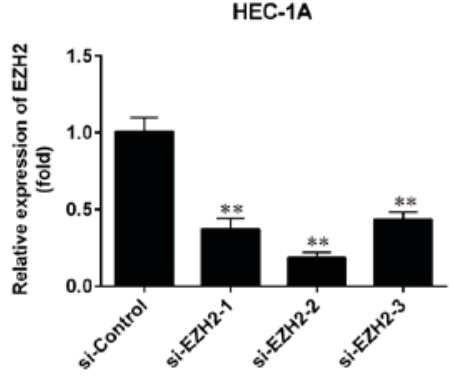

B

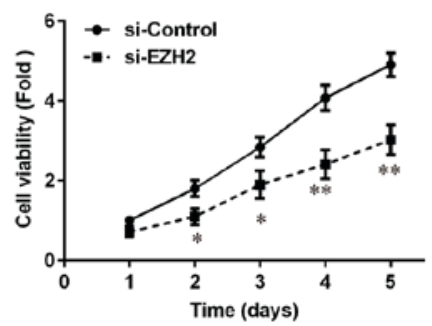

RL95-2

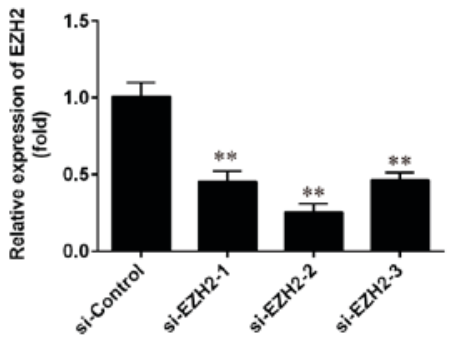

RL95-2

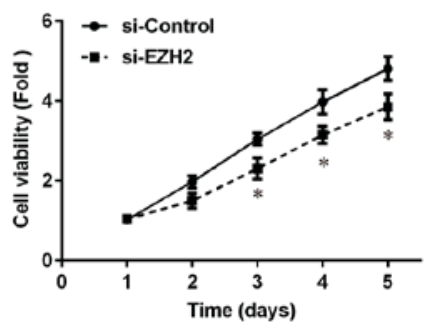

Figure 2. EZH2 knockdown inhibited cell proliferation. (A) The endometrial cancer cell HEC-1A and RL95-2 were transfected with three separated EZH2 siRNA or control siRNA respectively. The efficiency of EZH2 knockdown was examined by RT-qPCR. (B) Curves of cell viability were showed 24, 48, 72, 96, $120 \mathrm{~h}$ after EZH2-siRNA transfection by CCK8 assay. A significant decrease in proliferation was observed. Results shown are from three independent experiments. "P $<0.05 ;{ }^{* *} \mathrm{P}<0.01$ vs. the control group. EZH2, enhancer of zeste homolog 2; RT-qPCR, quantitative reverse transcription-polymerase chain reaction; si, small interfering.

A
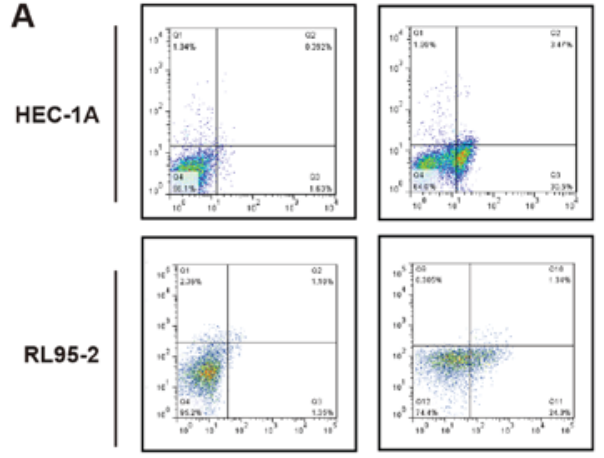

si-Control

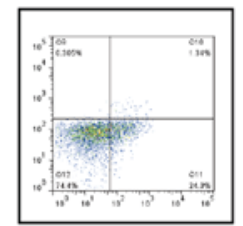

si-EZH2

B

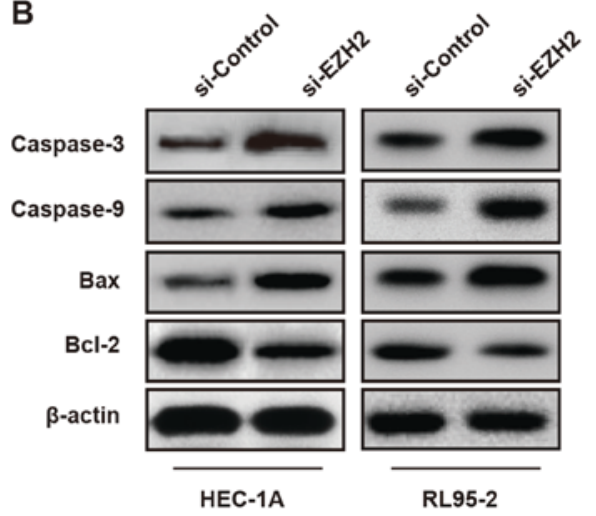

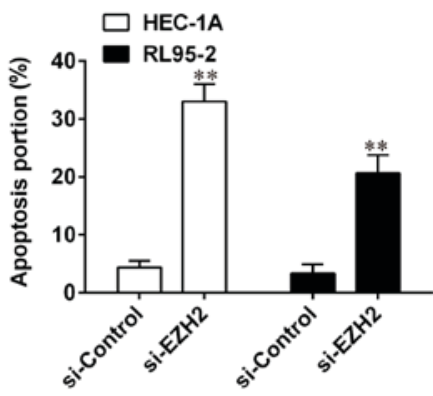

HEC-1A
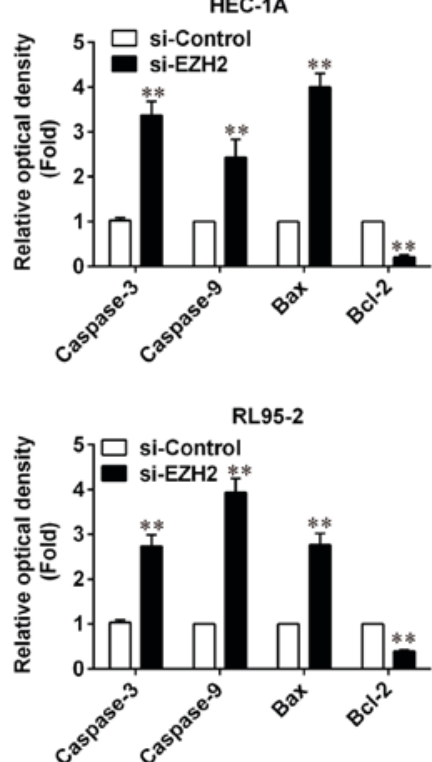

Figure 3. EZH2 knockdown increased cell apoptosis. (A) Induction of apoptosis was examined by Annexin V/PI double staining using a FACS analysis. (B) The marker proteins of apoptosis were detected by western blot analysis and RT-qPCR. The promoting apoptosis protein was increased and the anti-apoptosis protein was decreased. Results shown are from 3 independent experiments. ${ }^{* *} \mathrm{P}<0.01$ vs. the control group. EZH2, enhancer of zeste homolog 2; RT-qPCR, quantitative reverse transcription-polymerase chain reaction; PI, propidium iodide; Bax, BCL2 associated X. 

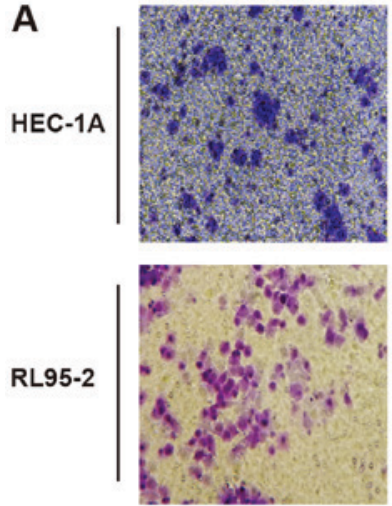

si-Control

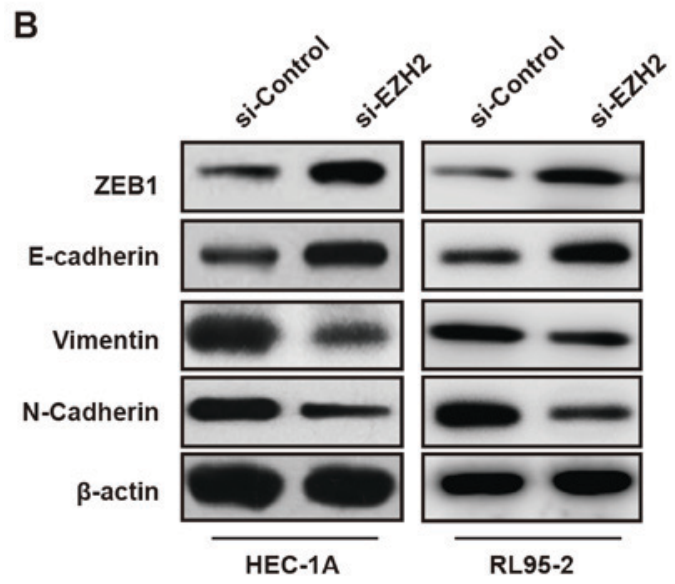

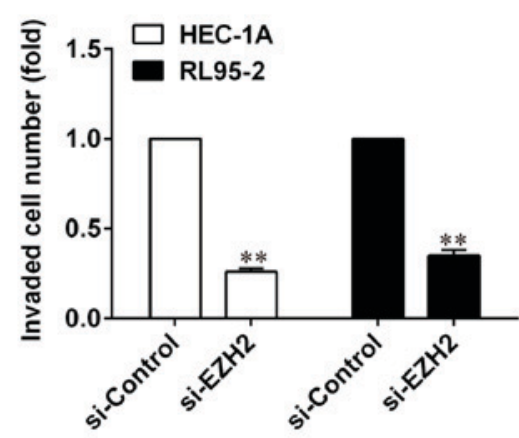

HEC-1A

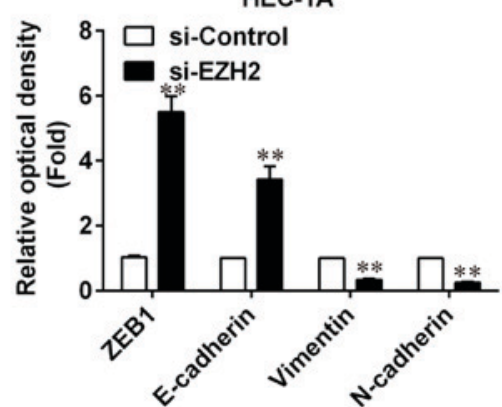

RL95-2

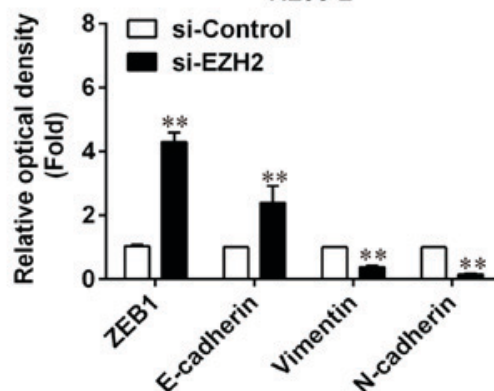

Figure 4. EZH2 knockdown inhibited the endometrial cancer cells invasion. (A) si-EZH2 and si-control cell lines were assayed for invasion by control and Matrigel-coated Transwell. The results were quantified by manual counting. ${ }^{* *} \mathrm{P}<0.01$ vs. the si-control group. (B) The markers of epithelial-mesenchymal transition were detected by western blot analysis (left) and RT-qPCR (right). A representative blot was shown from 3 independent experiments. EZH2, enhancer of zeste homolog 2; RT-qPCR, quantitative reverse transcription-polymerase chain reaction; si, small interfering; E-, epithelial-; N-, neural-; ZEB, zinc finger E-box binding homeobox.

EZH2 knockdown increases the apoptosis of human EC cells in vitro. The present study sought to determine the mechanisms by which EZH2 knockdown inhibits the growth of human EC cells. Annexin V positively stained cells determined by FACS showed that there was statistical difference between HEC-1A and RL95-2 cells transfected with siEZH2 and respective control (Fig. 3A). We next examined the markers of apoptosis in HEC-1A and RL95-2 cells transfected with siEZH2 compared with respective control. When compared with controls, markers of pro-apoptosis were significantly induced and anti-apoptosis $\mathrm{Bcl} 2$ protein was significantly decreased by siEZH2 (Fig. 3B). Taken together, it was concluded that EZH2 knockdown induces apoptosis of human EC cells.

EZH2 knockdown inhibits the invasion of endometrial cancer cells. EZH2 has been associated with regulating cell invasion and migration in numerous cancer tissues and cell lines, such as ovarian carcinoma cell (19), glioblastoma cells (20), and bladder cancer cells (21). Thus, we strived to examine the effects of EZH2 knockdown on invasion of human EC cells (HEC-1A and RL95-2). Towards this goal, HEC-1A and RL95-2 cells transfected with siEZH2 and respective control were tested for their ability to migrate through uncoated control membrane or invade through Matrigel-coated membrane. Compared with controls, EZH2 knockdown significantly inhibited the invasion of HEC-1A and RL95-2 cells as revealed by a decreased invasion index that is calculated as the ratio between the number of cells invaded through Matrigel-coated membrane and the number of cells migrated through control membrane (Fig. 4A). The epithelial-mesenchymal transition (EMT) has been implicated in cell invasion in multiple types of cancer cell lines, including breast cancer (22), colorectal cancer (23), and gastric cancer (24). Therefore, the present study further explored the association between the expression of EMT markers and EZH2 in the endometrial cancer cells by western blot analysis and RT-qPCR. As shown in Fig. 4B, EZH2 knockdown was associated with decreased vimentin and $\mathrm{N}$-cadherin and increased E-cadherin, suggesting EZH2 may influence the invasion of EC cells through promoting EMT. 


\section{Discussion}

As a transcriptional repressor, EZH2 plays a critical role in the control of cell proliferation, determination of stem cell fate and carcinogenesis (25). In addition, EZH2 has been identified as a prognostic marker for breast, ovarian, colon, gastric, oral, prostate cancer and melanoma (26). The present study demonstrated upregulated EZH2 expression in EC tissues and cell lines. The present study assessed the role of EZH2 in the biological behavior of EC through silencing EZH2. Taken together, these data indicated a significant role for targeting EZH2 in the influences of the development and progression in EC.

Multiple genes and microRNA (miR) have been associated with EZH2 inhibition-induced apoptosis (27,28). For instance, EZH2 knockdown induces apoptosis through the overexpression of FBX032 in breast cancer cells (29), abnormal expression of Bim and proapoptotic miR-31 as well as miR205 in prostate cancer cells and hepatocellular carcinoma (30-32). In the present study, it was found that EZH2 knockdown significantly increased the apoptosis in EC cells. The present study further examined the mechanism of EZH2 inhibition induced apoptosis and found markers of pro-apoptosis were significantly induced and anti-apoptosis $\mathrm{Bcl} 2$ protein was significantly reduced by siEZH 2 .

The tumor suppressor p53 and the transcriptional repressor EZH2 have been associated with the regulation of epithelial-mesenchymal transition (EMT) and tumor metastasis (33). Inhibition of EZH2 expression and upregulation of E-cadherin expression decrease the EMT process in vitro and in vivo (34). The EZH2 polycomb group protein drives an aggressive phenotype in melanoma cancer stem cells and is a target of diet derived sulforaphane (35). EZH2 is associated with poor prognosis in head-and-neck squamous cell carcinoma via regulating the epithelial-to-mesenchymal transition (36). Consistently, the results of the present study further confirmed the effect of EZH2 inhibition on the EMT by upregulation of E-cadherin and downregulation of $\mathrm{N}$-cadherin as well as vimentin, with an associated decrease in invasion.

There are limitations to the present study. First, the regulation effect of silencing EZH2 in vivo should be further explored. Second, the precise molecular mechanisms of EZH2 on apoptosis and EMT in EC have not been clearly clarified to date. Finally, future studies are required to further elucidate the comprehensive mechanisms for the influence of EZH2 in the development of EC and it was considered important for the forming of targeted treatment strategy in patients with EC.

To conclude, the data reported here revealed that EZH2 is overexpressed in ECs and its overexpression is associated with proliferation, apoptosis, invasion and EMT of EC cells in vitro. A comprehensive study of epigenetic mechanisms and the relevance of EZH2 in EC are important for fully understanding this disease and as a basis for developing novel treatment options in patients with EC.

\section{References}

1. Arnold M, Pandeya N, Byrnes G, Renehan PAG, Stevens GA, Ezzati PM, Ferlay J, Miranda JJ, Romieu I, Dikshit R, et al: Global burden of cancer attributable to high body-mass index in 2012: A population-based study. Lancet Oncol 16: 36-46, 2015.
2. Morice P, Leary A, Creutzberg C, Abu-Rustum N and Darai E: Endometrial cancer. Lancet 387: 1094-1108, 2016.

3. Arenas M, Gascón M, Rovirosa À, Hernández V, Riu F, López I, Montero A and Sabater S: The effect of lymphadenectomy and radiotherapy on recurrence and survival in endometrial carcinoma. Experience in a population reference centre. Rep Pract Oncol Radiother 20: 50-56, 2015.

4. Iavazzo C, Gkegkes ID and Vrachnis N: Early recurrence of early stage endometrioid endometrial carcinoma: Possible etiologic pathways and management options. Maturitas 78: 155-159, 2014.

5. Yoo KH and Hennighausen L: EZH2 methyltransferase and H3K27 methylation in breast cancer. Int J Biol Sci 8: 59-65, 2012.

6. Li LY: EZH2: Novel therapeutic target for human cancer. Biomedicine (Taipei) 4: 1, 2014.

7. $\mathrm{Xu} \mathrm{K}, \mathrm{Wu}$ ZJ, Groner AC, He HH, Cai C, Lis RT, Wu X, Stack EC, Loda M, Liu T, et al: EZH2 oncogenic activity in castration-resistant prostate cancercells is Polycomb-independent. Science 338: 1465-1469, 2012.

8. Curry E, Green I, Chapman-Rothe N, Shamsaei E, Kandil S, Cherblanc FL, Payne L, Bell E, Ganesh T, Srimongkolpithak N, et al: Dual EZH2 and EHMT2 histone methyltransferase inhibition increases biological efficacy in breast cancer cells. Clin Epigenetics 7: 84, 2015.

9. Zhang YB, Niu HT, Chang JW, Dong GL and Ma XB: EZH2 silencing by RNA interference inhibits proliferation in bladder cancer cell lines. Eur J Cancer Care (Engl) 20: 106-112, 2011.

10. Behrens C, Solis LM, Lin H, Yuan P, Tang X, Kadara H, Riquelme E, Galindo H, Moran CA, Kalhor N, et al: EZH2 protein expression associates with the early pathogenesis, tumor progression, and prognosis of non-small cell lung carcinoma. Clin Cancer Res 19: 6556-6565, 2013.

11. Li H and Zhang R: Role of EZH2 in epithelial ovarian cancer: From biological insights to therapeutic target. Front Oncol 3: 47, 2013.

12. Bachmann IM, Halvorsen OJ, Collett K, Stefansson IM, Straume O, Haukaas SA, Salvesen HB, Otte AP and Akslen LA: EZH2 expression is associated with high proliferation rate and aggressive tumor subgroups in cutaneous melanoma and cancers of the endometrium, prostate, and breast. J Clin Oncol 24: 268-273, 2006.

13. Mannelqvist M, Stefansson IM, Wik E, Kusonmano K Raeder MB, Øyan AM, Kalland KH, Moses MA, Salvesen HB and Akslen LA: Lipocalin 2 expression is associated with aggressive features of endometrial cancer. BMC Cancer 12: 169, 2012.

14. Zhou J, Roh JW, Bandyopadhyay S, Chen Z, Munkarah AR, Hussein Y, Alosh B, Jazaerly T, Hayek K, Semaan A, et al: Overexpression of enhancer of zeste homolog 2 (EZH2) and focal adhesion kinase (FAK) in high grade endometrial carcinoma. Gynecol Oncol 128: 344-348, 2013.

15. Eskander RN, Ji T, Huynh B, Wardeh R, Randall LM and Hoang B: Inhibition of enhancer of zeste homolog 2 (EZH2) expression is associated with decreased tumor cell proliferation, migration, and invasion in endometrial cancer cell lines. Int J Gynecol Cancer 23: 997-1005, 2013.

16. Jia N, Li Q, Tao X, Wang J, Hua K and Feng W: Enhancer of zeste homolog 2 is involved in the proliferation of endometrial carcinoma. Oncol Lett 8: 2049-2054, 2014.

17. Livak KJ and Schmittgen TD: Analysis of relative gene expression data using real-time quantitative PCR and the 2(-Delta Delta C(T)) method. Methods 25: 402-408, 2001.

18. Li H, Cai Q, Godwin AK and Zhang R: Enhancer of zeste homolog 2 promotes the proliferation and invasion of epithelial ovarian cancer cells. Mol Cancer Res 8: 1610-1618, 2010.

19. Rao ZY, Cai MY, Yang GF, He LR, Mai SJ, Hua WF, Liao YJ, Deng HX, Chen YC, Guan XY, et al: EZH2 supports ovarian carcinoma cell invasion and/or metastasis via regulation of TGF-beta1 and is a predictor of outcome in ovarian carcinoma patients. Carcinogenesis 31: 1576-1583, 2010.

20. Smits M, Nilsson J, Mir SE, van der Stoop PM, Hulleman E, Niers JM, de Witt Hamer PC, Marquez VE, Cloos J, Krichevsky AM, et al: miR-101 is down-regulated in glioblastoma resulting in EZH2-induced proliferation, migration, and angiogenesis. Oncotarget 1: 710-720, 2010.

21. Luo M, Li Z, Wang W, Zeng Y, Liu Z and Qiu J: Long non-coding RNA H19 increases bladder cancer metastasis by associating with EZH2 and inhibiting E-cadherin expression. Cancer Lett 333: 213-221, 2013

22. Ma L, Teruya-Feldstein $\mathrm{J}$ and Weinberg RA: Tumour invasion and metastasis initiated by microRNA-10b in breast cancer. Nature 449: 682-688, 2007. 
23. Spaderna S, Schmalhofer O, Hlubek F, Berx G, Eger A, Merkel S, Jung A, Kirchner T and Brabletz T: A transient, EMT-linked loss of basement membranes indicates metastasis and poor survival in colorectal cancer. Gastroenterology 131: 830-840, 2006.

24. Yang Z, Zhang X, Gang H, Li X, Li Z, Wang T, Han J, Luo T, Wen $\mathrm{F}$ and $\mathrm{Wu} \mathrm{X}$ : Up-regulation of gastric cancer cell invasion by twist is accompanied by $\mathrm{N}$-cadherin and fibronectin expression. Biochem Biophys Res Commun 358: 925-930, 2007.

25. Simon JA and Lange CA: Roles of the EZH2 histone methyltransferase in cancer epigenetics. Mutat Res 647: 21-29, 2008.

26. Völkel P, Dupret B, Le Bourhis X and Angrand PO: Diverse involvement of EZH2 in cancer epigenetics. Am J Transl Res 7: 175-193, 2015

27. Zhang B, Liu XX, He JR, Zhou CX, Guo M, He M, Li MF, Chen GQ and Zhao Q: Pathologically decreased miR-26a antagonizes apoptosis and facilitates carcinogenesis by targeting MTDH and EZH2 in breast cancer. Carcinogenesis 32: 2-9, 2011.

28. Wu ZL, Zheng SS, Li ZM, Qiao YY, Aau MY and Yu Q: Polycomb protein EZH2 regulates E2F1-dependent apoptosis through epigenetically modulating Bim expression. Cell Death Differ 17: 801-810, 2010.

29. Salazar H, Godwin AK, Daly MB, Laub PB, Hogan WM Rosenblum N, Boente MP, Lynch HT and Hamilton TC: Microscopic benign and invasive malignant neoplasms and a cancer-prone phenotype in prophylactic oophorectomies. J Natl Cancer Inst 88: 1810-1820, 1996.

30. Wu ZL, Zheng SS, Li ZM, Qiao YY, Aau MY and Yu Q: Polycomb protein EZH2 regulates E2F1-dependent apoptosis through epigenetically modulating Bim expression. Cell Death Differ 17: 801-810, 2010.
31. Zhang Q, Padi SK, Tindall DJ and Guo B: Polycomb protein EZH2 suppresses apoptosis by silencing the proapoptotic miR-31. Cell Death Dis 5: e1486, 2014.

32. Yang F, Lv LZ, Cai QC and Jiang Y: Potential roles of EZH2, Bmi-1 and miR-203 in cell proliferation and invasion in hepatocellular carcinoma cell line Hep3B. World J Gastroenterol 21: 13268-13276, 2015.

33. Jiang FZ, He YY, Wang HH, Zhang HL, Zhang J, Yan XF, Wang XJ, Che Q, Ke JQ, Chen Z, et al: Mutant p53 induces EZH2 expression and promotes epithelial-mesenchymal transition by disrupting p68-Drosha complex assembly and attenuating miR-26a processing. Oncotarget 6: 44660-44674, 2015.

34. Ma DN, Chai ZT, Zhu XD, Zhang N, Zhan DH, Ye BG, Wang CH, Qin CD, Zhao YM, Zhu WP, et al: MicroRNA-26a suppresses epithelial-mesenchymal transition in human hepatocellular carcinoma by repressing enhancer of zeste homolog 2 . J Hematol Oncol 9: 1,2016.

35. Fisher ML, Adhikary G, Grun D, Kaetzel DM and Eckert RL: The Ezh2 polycomb group protein drives an aggressive phenotype in melanoma cancer stem cells and is a target of diet derived sulforaphane. Mol Carcinog 55: 2024-2036, 2016.

36. Chang JW, Gwak SY, Shim GA, Liu L, Lim YC, Kim JM, Jung MG and Koo BS: EZH2 is associated with poor prognosis in head-and-neck squamous cell carcinoma via regulating the epithelial-to-mesenchymal transition and chemosensitivity. Oral Oncol 52: 66-74, 2016. 